\title{
QUÉ EMOCIONES PIENSAN LOS FUTUROS MAESTROS QUE SIENTEN LOS ALUMNOS ANTE EL CYBERBULLYING. ANÁLISIS DE SU EXPERIENCIA PREVIA SOBRE LA PERCEPCIÓN
}

\author{
Marta de las Heras Martínez \\ Departamento de Psicología Evolutiva y de la Educación \\ Universidad de Castilla-La Mancha \\ Marta.Heras@uclm.es
}

Fecha de Recepción: 8 Febrero 2019

Fecha de Admisión: 30 Abril 2019

\section{RESUMEN}

El escenario del futuro maestro nos muestra una población con escasa experiencia respecto a la forma de actuar frente al acoso (Álvarez-García et al, 2010). Muchos estudios se han centrado en el análisis de las emociones como factor de motivación del comportamiento. Los resultados han mostrado que actuar sobre las emociones puede provocar una modificación de conducta hacia la reducción del acoso. Las investigaciones señalan que bajos niveles de emociones negativas pueden legitimar los comportamientos de acoso (Perren y Gutzwiller-Helfenfinger, 2012) y que la experimentación de satisfacción puede contribuir a su incremento (Menesini, Palladino y Nocentini, 2015). Nuestro objetivo es conocer la percepción del cyberbullying en las aulas en los futuros maestros, y analizar si está condicionada por su experiencia previa con el acoso. Han participado en el estudio 1122 estudiantes del grado de Educación de la Universidad de Castilla-La Mancha. Los alumnos tenían que identificar qué emociones consideraban que caracterizaban a víctimas, agresores y observadores. La escala estaba constituida por 14 emociones, placenteras, displacenteras y neutras (Larrañaga, Navarro y Yubero, 2018), con respuesta dicotómica. Los resultados apuntan que los futuros maestros reconocen en las víctimas miedo, indefensión, malestar, tristeza y preocupación. Asocian el comportamiento agresivo con emociones positivas: satisfacción, diversión y bienestar. Es interesante destacar que en víctimas y observadores consideran que aparece la culpa y la vergüenza. En el análisis diferencial según la experiencia previa, los que han sido agresores informaron de mayor ira en las víctimas, ligeramente superior en el rol mixto de víctima/agresor. También en mayor porcentaje de culpa en los agresores y los observadores. Los estudiantes que habían sido víctimas/agresores indican en mayor porcentaje que los agresores no sienten 'nada'. Casi el 80\% de los que habían sido agresores indicaron que en los observadores se producía sorpresa. Estos resultados pueden tener implicaciones relevantes para la intervención en las aulas.

Palabras clave: futuros maestros; cyberbullying; emociones; experiencia previa 


\section{ABSTRACT}

What emotions think future teachers feel pupils before cyberbullying. Analysis of your previous experience about perception. El escenario del futuro maestro nos muestra una población con escasa experiencia respecto a la forma de actuar frente al acoso (Álvarez-García et al, 2010).The scenario of the future teacher shows us a population with Little experience regarding the way of acting against bullying (Álvarez-García et al, 2010) Many studies have focused on the analysis of emotions as a motivating factor of behavior. $L$ os resultados han mostrado que actuar sobre las emociones puede provocar una modificación de conducta hacia la reducción del acoso. The results have shown that acting on emotions can lead to behavior )modification towards reducing bullying. Las investigaciones señalan que $b$ ajos niveles de emociones negativas pueden legitimar los comportamientos de acoso ( Perren y Gutzwiller-Helfenfinger , 2012) y que la experimentación de satisfacción puede contribuir a su incremento ( Menesini , Palladino y Nocentini , 2015). Research indicates that high levels of negative emotions can legitimize bullying behaviors (Perren, \& Gutzwiller-Helfenfinger, 2012) and that satisfaction experimentation can contribute to their increase (Menesini, Palladino, \& Nocentini, 2015). Nuestro objetivo es conocer la percepción del cyberbullying en las aulas en los futuros maestros, y analizar si está condicionada por su experiencia previa con el acoso. Our goal is to know the perception of cyberbullying in the classrooms of future teachers, and analyze if it is conditioned by their previous experience with bullying. Han participado en el estudio 1122 estudiantes del grado de Educación de la Universidad de Castilla-La Mancha. Have participated in the study 1122 students of the degree of Education of the University of CastillaLa Mancha. Los alumnos tenían que identificar qué emociones consideraban que caracterizaban a víctimas, agresores y observadores. The students had to identify which emotions they considered to characterize victims, aggressors and observers. La escala estaba constituida por 14 emociones, placenteras, displacenteras y neutras ( Larrañaga , Navarro y Yubero , 2018), con respuesta dicotómica.The scale consisted of 14 emotions, pleasurable, unpleasant and neutral (Larrañaga, Navarro, \& Yubero, 2018), with a dichotomous response. Los resultados apuntan que los futuros maestros $r$ econocen en las víctimas miedo, indefensión, malestar, tristeza y preocupación. The results point out that the future teachers find in the victims fear, helplessness, discomfort, sadness and worry. Asocian el comportamiento agresivo con emociones positivas: satisfacción, diversión y bienestar. They associate aggressive behavior with positive emotions: satisfaction, fun and wellbeing. Es interesante destacar que en víctimas y observadores consideran que aparece la culpa y la vergüenza. It is interesting to note that victims and observers consider that guilt and shame appear. En el análisis diferencial según la experiencia previa, los que han sido agresores informaron de mayor ira en las víctimas, ligeramente superior en el rol mixto de víctima/agresor. In the differential analysis according to previous experience, those who have been aggressors reported greater anger in the victims, slightly higher in the mixed role of victim / aggressor. También en mayor porcentaje de culpa en los agresores y los observadores. Also in greater percentage of fault in the aggressors and the observers. Los estudiantes que habían sido víctimas/agresores indican en mayor porcentaje que los agresores no sienten 'nada'. The students who had been victims / aggressors indicate in a greater percentage that the aggressors do not feel 'nothing'. Casi el $80 \%$ de los que habían sido agresores indicaron que en los observadores se producía sorpresa. Almost $80 \%$ of those who had been aggressors indicated that surprise occurred in the observers. Estos resultados pueden tener implicaciones relevantes para la intervención en las aulas. These results may have relevant implications for the intervention in the classrooms.

Keywords : future teachers; cyberbullying; emotions; previous experience 


\section{INTRODUCCIÓN}

Durante la última década se han incrementado los episodios de acoso haciendo uso de las nuevas tecnologías de la comunicación (Smith, Mahdavi, Carvalho, Fisher, Russell y Tippett, 2008). El cyberbullying ha sido definido como un acto agresivo deliberado y repetido que es infringido a través de ordenadores, móviles u otros aparatos electrónicos (Hinduja y Patchin, 2015), sobre una víctima que no puede defenderse por sí misma (Smith et al, 2008). Ambas definiciones enfatizan la naturaleza digital de la conducta agresiva y se refieren a conductas que son intencionadas, que se prolonga en el tiempo y que producen un daño a quien las sufren (Larrañaga, Navarro y Yubero, 2018). La literatura científica evidencia un aumento significativo en la última década del cyberbullying (Brochado, Soares, \& Fraga, 2017; Selkie, Fales, \& Moreno, 2016), situando la prevalencia media de victimas entre el 20 y el 30\% (Garaigordobil, 2011).

El escenario del futuro maestro nos muestra una población con escasa experiencia, en el momento de realizar sus prácticas en los centros educativos, respecto a la forma de actuar frente al acoso (Álvarez-García et al, 2010). Muchos estudios se han centrado en el análisis de las emociones como factor de motivación del comportamiento del bullying y del cyberbullying. Los resultados han mostrado que actuar sobre las emociones puede provocar una modificación de conducta hacia la reducción del acoso. Las investigaciones señalan que bajos niveles de emociones negativas pueden legitimar los comportamientos de acoso (Perren y Gutzwiller-Helfenfinger, 2012). Si, además, los agresores experimentan satisfacción por su conducta puede contribuir al incremento de su comportamiento (Menesini, Palladino y Nocentini, 2015).

\section{OBJETIVO}

En este contexto, nuestro objetivo es conocer la percepción del cyberbullying en las aulas de los futuros maestros, y analizar si está condicionada por su experiencia previa en situaciones de acoso. Se trata de conectar las variables experienciales, según haya sido el papel adoptado en las dinámicas de bullying y cyberbullying, en forma de agresor, víctima, víctima/agresor o no interviniendo, con las creencias actuales como futuro docente sobre las emociones experimentadas por víctimas, agresores y observadores.

\section{MÉTODO}

\section{Participantes}

La muestra está formada por 1122 estudiantes del Grado de Educación de la Universidad de Castilla-La Mancha, un 78.3\% son mujeres; un 48.9\% estudian el Grado de Educación Infantil y un $51.1 \%$ el de Educación Primaria.

\section{Instrumentos}

Para evaluar el componente emocional de los participantes en el cyberbullying se ha empleado una adaptación de la escala de la investigación de Larrañaga, Navarro y Yubero (2018). Los alumnos tenían que identificar qué emociones consideraban que caracterizaban a víctimas, agresores y observadores. La escala está constituida por 14 emociones, placenteras, displacenteras y neutras, con respuesta dicotómica.

\section{Procedimiento}

El cuestionario fue pasado en las aulas de las distintas Facultades de Educación que conforman la Universidad de Castilla-La Mancha, previo acuerdo con los Decanos y profesores. Se explicó a los estudiantes del Grado de Educación el objetivo del estudio y se informó sobre la voluntariedad de 
su participación y anonimato de sus respuestas. El tiempo medio aproximado de cumplimentación fue de 10 minutos.

\section{Análisis de datos}

El análisis de los datos cuantitativos se realizó con el programa SPSS-24. En primer lugar, se realizó un análisis descriptivo de las emociones que percibían los futuros maestros según el rol de intervención de los alumnos en el acoso. Con posterioridad, se realizó un análisis inferencial con Anovas según la experiencia previa de intervención en el acoso que los futuros maestros informaron en los niveles previos educativos.

\section{RESULTADOS}

El primer nivel de análisis se centró en valorar la experiencia previa en situaciones de acoso por parte de los futuros maestros: Un $28.3 \%$ había sido victimizado durante su escolarización, un $4.4 \%$ se reconoció agresor, la mayoría, un 40,2\% había intervenido como víctima y agresor y sólo un $27.1 \%$ no había participado en el acoso (gráfica 1).

Gráfica 1: Experiencia previa en el acoso

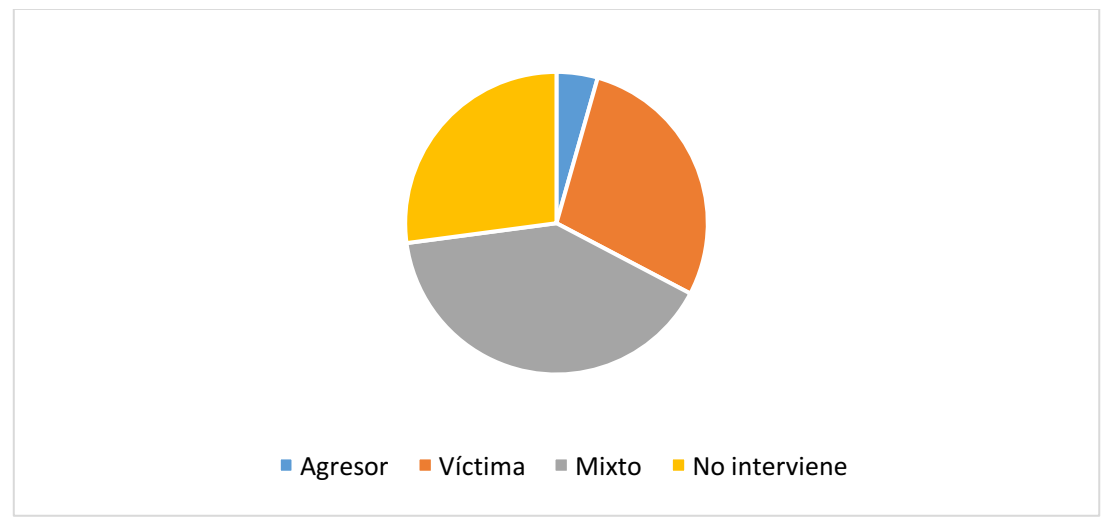

Por lo que se refiere a las creencias, en cuanto a las emociones sentidas por los tres roles implicados en las dinámicas de acoso, los futuros docentes reconocen en las víctimas miedo, indefensión, malestar, tristeza y preocupación. Asocian el comportamiento agresivo con emociones positivas: satisfacción, diversión y bienestar. Es interesante destacar que en víctimas y observadores consideran que aparece la culpa y la vergüenza (tabla 1) 
Tabla 1: Emociones sentidas

\begin{tabular}{|l|l|l|}
\hline Víctimas & Agresores & Observadores \\
\hline Miedo (96.7) & Satisfacción (90.7) & Compasión (78.6) \\
Indefensión (92.8) & Diversión (88.7) & Preocupación (72.7) \\
Malestar (91.1) & Bienestar (78.4) & Sorpresa (58.7) \\
Tristeza (84.4) & Ira (62.3) & Tristeza (53.0) \\
Vergüenza (77.4) & Nada (35.6) & Culpa (47.1) \\
Culpa (60.4) & & Vergüenza (46.3) \\
Preocupación (58.4) & & Malestar (42.8) \\
\hline
\end{tabular}

En el análisis diferencial, según la experiencia previa, los que han sido agresores informaron de mayor ira en las víctimas, ligeramente superior en el rol mixto de víctima/agresor. También en mayor porcentaje de culpa en los agresores y los observadores. Los estudiantes que habían sido víctimas/agresores indican en mayor porcentaje que los agresores no sienten 'nada'. Casi el 80\% de los que habían sido agresores indicaron que en los observadores se producía sorpresa (tabla 2).

Tabla 2: Emociones diferenciales sentidas según experiencia previa

\begin{tabular}{|c|c|c|c|c|c|}
\hline & $\begin{array}{c}\text { No } \\
\text { Interviene }\end{array}$ & Víctima & Agresor & $\begin{array}{l}\text { Víctima/ } \\
\text { Agresor }\end{array}$ & $\chi^{2}$ \\
\hline \multicolumn{6}{|c|}{ ¿Qué crees que sienten las víctimas? (\%) } \\
\hline Ira & 31.9 & 37.7 & 44.9 & 46.2 & $16.52 * * *$ \\
\hline \multicolumn{6}{|c|}{ ¿Qué crees que sienten los agresores? (\%) } \\
\hline Culpa & 16.4 & 15.3 & 22.4 & 22.3 & $7.63 *$ \\
\hline Nada & 33.7 & 31.1 & 31.3 & 40.5 & $7.99 *$ \\
\hline \multicolumn{6}{|c|}{ ¿Qué crees que sienten los observadores? (\%) } \\
\hline Sorpresa & 52.3 & 58.8 & 77.6 & 62.4 & $15.09 * *$ \\
\hline Culpa & 37.6 & 48.1 & 51.0 & 51.9 & $15.35 * *$ \\
\hline
\end{tabular}

Nota: ${ }^{*} p<.05, * * p<.01, * * * p<.001$

\section{DISCUSIÓN Y CONCLUSIONES}

A la vista de los resultados podemos afirmar que los futuros maestros reconocen que los agresores desestiman el impacto de su comportamiento en las víctimas, lo que puede llevar a sentirse satisfechos con su comportamiento. Es uno de los factores importantes de intervención a nivel motivacional en las aulas. Es imprescindible que los agresores reconozcan el daño que causa su comportamiento sobre los compañeros. 
Por otra parte, la asunción de culpa y vergüenza por las víctimas y los observadores debe ser también foco de intervención. En las víctimas es fundamental eliminar esos sentimientos para que puedan pedir ayuda libremente. En los observadores hay que trabajar para que esas emociones les lleven a la toma de decisión de ayuda de sus compañeros víctimas del acoso. El reconocimiento de estas emociones es un paso importante para conseguir un rol activo por parte de los educadores.

Aún así, se contempla la necesidad de que la Universidad, como centro de formación inicial de los maestros, incremente la formación respecto al bullying y el cyberbullying (Calatayud, Prendes y López, 2016). La investigación llevada a cabo por Ríoseco y Menéndez (2017), con una muestra de futuros docentes, apoya este aspecto y ve necesario su abordaje en la escuela a través de políticas gubernamentales, confirmando que muchos de ellos no se sienten preparados para detectar ni abordar dichas situaciones.

\section{REFERENCIAS BIBLIOGRÁFICAS}

Álvarez-Ga ${ }^{a}$, D. et at (2010). La formación de los futuros docents frente a la violencia escolar. Revista de Psicodidáctica, 15 (1), 35-36

González-Calatayud, V., Prendes, M.P., y Lopez, J.A., (2016). La percepción sobre el ciberacoso del profesorado de secundaria en la Región de Murcia. International Journal of Technology and Educational Innovation, 2 (1), 84-89

Hinduja, S., \& Patchin, J. W. (2015). Bullying Beyond the Schoolyard: Preventing and Responding to Cyberbullying (2nd edition). Thousand Oaks, CA: Sage Publications.

Larrañaga, E., Navarro, R., \& Yubero, S. (2018). Factores socio-cognitivos y emocionales en la agresión del ciberacoso. Comunicar, 56, 19-28. doi: https://doi.org/10.3916/C56-2018-02

Menesini, E., Palladino, B.E., \& Nocentini, A. (2015). Emotions of moral disengagement, class norms, and bullying in adolescence: A multilevel approach. Merrill-Palmer Quarterly, 61(1), 124143. doi: https://doi.org/10.13110/merrpalmquar1982.61.1.0124

Ortega-Barón \& Carrascosa (2018). Malestar psicológico y apoyo psicosocial en víctimas de cyberbullying. International Journal of Developmental and Educational Psychology INFAD Revista de Psicología, №1 - Monográfico 1, 2018. ISSN: 0214-9877. pp:357-366

Perren, S., \& Gutzwiller-Helfenfinger, E. (2012). Cyberbullying and traditional bullying in adolescence: Differential roles of moral disengagement, moral emotions, and moral values. European Journal of Developmental Psychology, 9, 195-209. doi: https://doi.org/10.1080/17405629.2011.643168

Rioseco, M., \& Meléndez, D. (2017). Ciberbullying from the perspective of initial of teacher trainees. Revista Dilemas Contemporáneos: Educación, Política y Vvalores, 2(19).

Smith, P., Mahdavi, J., Carvalho, M., Fisher, S., Russell. S., \& Tippett, N. (2008). Cyberbullying: Its nature and impact in secondary school pupils. Journal of Child Psychology and Psychiatry, 49(4), 376-385. doi: 